Legrain, G., Moes, N. and Huerta, A., Étude de la stabilité d'une formulation incompressible traitée par X-FEM, European Journal of Computational

Mechanics (Revue Européenne de Mécanique Numérique), Vol. 15, Issues 1, 2, 3, pp. 257-268, 2006

\title{
Etude de la stabilité d'une formulation incompressible traitée par X-FEM
}

\author{
Gregory Legrain* — Nicolas Moës* - Antonio Huerta** \\ * GeM - Institut de Recherche en Genie Civil et Mécanique \\ École Centrale de Nantes - Université de Nantes - CNRS UMR 6183 \\ 1, rue de la Nö̈, BP 92101 - F-44321 Nantes Cedex 3 \\ ** LaCàN - Laboratori de Càlcul Numèric - Edifici C2, Campus Nord, \\ Universitat Politècnica de Catalunya - E-08034 Barcelona, Spain
}

\begin{abstract}
RÉSUMÉ. Le traitement de l'incompressibilité est un point-clé pour le dimensionnement des composants élastomères ou la simulation du processus de formage. L'utilisation de formulations mixtes permet d'éviter le phénomène de verrouillage (locking) de l'approximation éléments finis. Cependant, la stabilité de ces méthodes est conditionnée par la vérification de la condition inf-sup. Récemment, les approximations E.F. ont évolué avec l'introduction de la partition de l'unité. La méthode X-FEM (eXtended Finite Element Method) utilise ce concept pour éviter le maillage (et remaillage) des surfaces physiques du problème. Dans cet article, une stratégie est proposée pour la gestion des trous avec la méthode X-FEM dans le cas incompressible. Les applications numériques montrent que la convergence théorique des éléments finis est préservée, et que la condition inf-sup est vérifiée.

ABSTRACT. The treatment of (near-)incompressibility is a major concern for the simulation of rubber-like parts, or forming processes. The use of mixed finite element methods is known to prevent the locking of the F.E. approximation in the incompressible limit. However, the stability of these formulations is conditionned by the fullfilment of the inf-sup condition. Recently, finite elements method has evolved with the introduction of the partition of unity. The X-FEM uses it to remove the need to mesh (and remesh) physical surfaces. In this paper, a strategy is proposed for the treatment of holes within X-FEM in the incompressible setting. Numerical examples show that F.E. convergence rate is preserved and that the inf-sup condition is passed.

MOTS-CLÉS : formulation mixte, X-FEM, partition de l'unité, condition inf-sup, incompressibilité, trous

KEYWORDS: mixed formulation, X-FEM, partition of unity, inf-sup condition, incompressibility, holes
\end{abstract}




\section{Introduction}

Les formulations éléments finis en déplacement sont largement utilisées pour les simulations en ingénierie. En effet, elles permettent la résolution d'une grande variété de problèmes, et sont maintenant bien connues sur le plan mathématique. Cependant, il existe encore deux limitations à ces méthodes. D'abord, la résolution de problèmes incompressibles ou quasi incompressibles qui nécessitent l'utilisation de formulations adaptées. Sinon, la contrainte d'incompressibilité verrouille le maillage (locking), donnant lieu à des solutions non physiques. En outre, la génération et surtout la réactualisation du maillage dans les cas 3D complexes faisant intervenir des frontières mobiles (fissures, interfaces matériau, vides) manque toujours de robustesse.
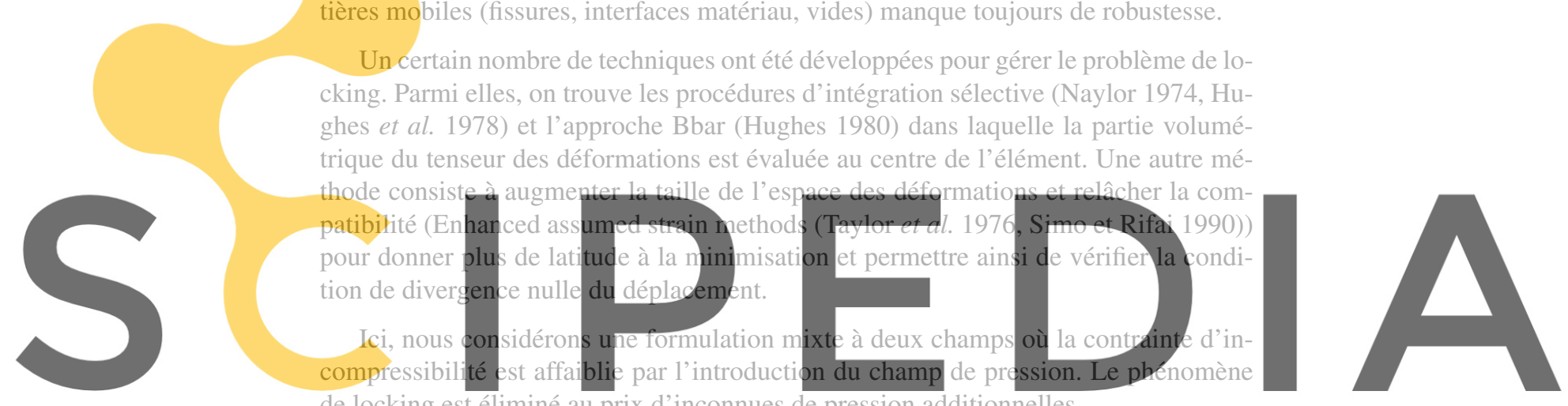

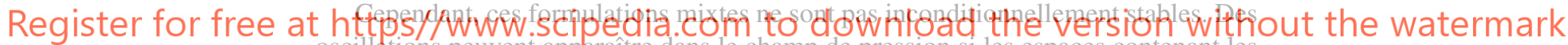 oscillations peuvent apparaître dans le champ de pression si les espaces contenant les déplacements et la pression ne sont pas choisis avec attention.}

Pour être stable, une formulation mixte doit vérifier la condition dite inf-sup (ou LBB). Cette dernière est une condition sévère qui dépend du lien entre les espaces d'approximation de déplacement et de pression. Prouver qu'une paire d'espaces déplacement-pression vérifie la condition inf-sup n'est pas une tâche aisée. Cependant, un test numérique a été proposé par Brezzi et Fortin (1991) puis par Chapelle et Bathe (1993), afin de prédire la vérification de la LBB.

La seconde limitation des formulations éléments finis classiques (frontières mobiles) a été résolue par le développement de méthodes alternatives comme les méthodes sans maillage avec lesquelles la connectivité entre les nœuds n'est plus obtenue par un maillage, mais par des domaines d'influence qui peuvent être coupés par les frontières. Cependant, ces méthodes sont sensibles au phénomène de verrouillage (Huerta et Fernández-Méndez 2001, Dolbow et Belytschko 1999) au même titre que les éléments finis. C'est pourquoi des stratégies ont été développées pour résoudre ce problème (Huerta et Fernández-Méndez 2001, Huerta et al. 2004). A part le couplage de méthode sans maillage avec les éléments finis (Huerta et FernándezMéndez 2000), la méthode X-FEM (eXtended Finite Element Method) est une alternative au remaillage. Cette méthode est basée sur la partition de l'unité (Melenk et 
Babuška 1996). Un enrichissement adapté de la base éléments finis permet de modéliser des trous, des inclusions matérielles et des fissures avec des maillages non conformes. La méthode X-FEM a été utilisée pour la simulation d'une grande variété de problèmes en mécanique de la rupture : fissuration 2D (Moës et al. 1999), 3D (Moës et al. 2002b, Gravouil et al. 2002), plaques (Dolbow et al. 2000), rupture cohésive (Moës et Belytschko 2002a), fissuration dynamique (Réthoré et al. 2005), fissuration non-linéaire (Dolbow et Devan 2004, Legrain et al. 2005b, Areias et Belytschko 2005). La gestion des trous a été étudiée dans (Daux et al. 2000), 1es inclusions matérielles dans (Sukumar et al. 2001, Moës et al. 2003) et les écoulements de phases multiples dans (Chessa et al. 2003).

Ici, nous nous intéressons à l'application de cette méthode pour le traitement des trous dans les matériaux incompressibles. Les méthodes de type Bbar et intégration réduite ne sont pas considérées, car elles ne semblent pas pouvoir être généralisées aisément aux champs de déplacement enrichis.

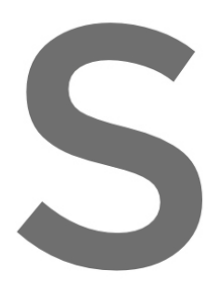

Dans cet article, on s'intéressera à la stabilité de formulations mixtes dans le cadre

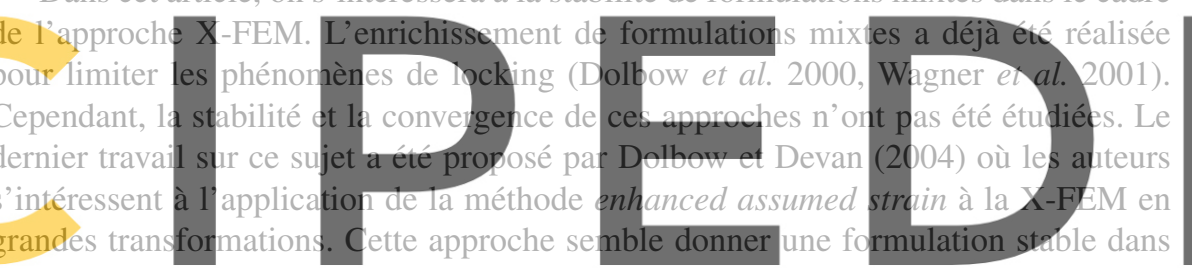

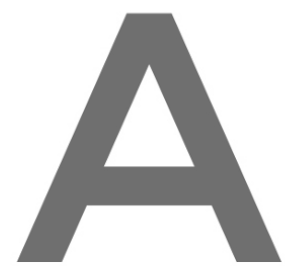

le cas de la mécanique non linéaire de la rupture pour des matériaux quasi incompressibles. Cependant, la stạbilité de la méthode n'a pas été étudiée en détail. Register for free at https//www.scipedia.com to download the version without the watermark

ticité linéaire incompressible sont rappelées. Ensuite, les conditions de stabilité des formulations mixtes sont présentées. Enfin, la prise en compte des trous dans le cas de formulations incompressibles traitées par X-FEM est présentée, puis validée.

\section{2. Équations du problème}

Dans cette section, on s'intéresse à la construction de formulations mixtes stables pour la résolution de problèmes d'élasticité incompressible. Les équations gouvernant le problème sont d'abord rappelées. Ensuite, la condition de compatibilité inf-sup ainsi qu'un test numérique sont présentés.

\section{1. Élasticité incompressible}

On considère la réponse statique d'un solide élastique occupant un domaine borné $\Omega \in \mathbb{R}^{2}$ avec des frontières suffisamment régulières $\partial \Omega$ qui sont séparées en deux parties disjointes : $\partial \Omega_{u}$ où les déplacements sont imposés et $\partial \Omega_{T}$ où le vecteur traction 


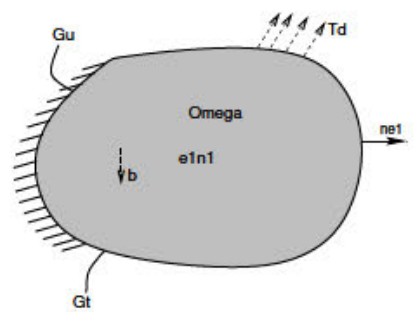

Figure 1. Le problème de référence

est imposé. Les équations gouvernant le problème d'élasticité linéaire bidimensionnel s'écrivent :
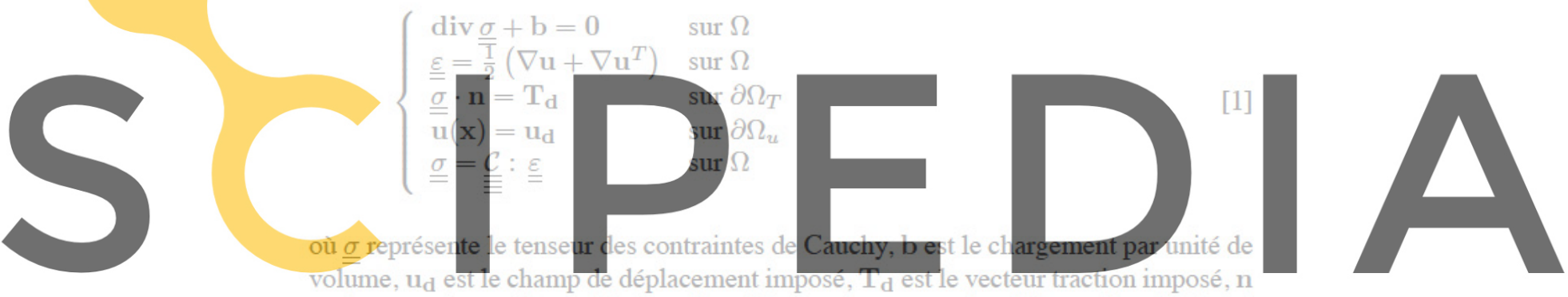

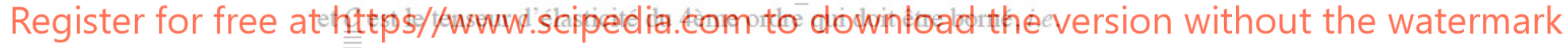

$$
C_{i j k l} \in L^{\infty}(\Omega) \quad i, j, k, l=1,2,3
$$

Dans le cas de l'élasticité linéaire isotrope, la relation de comportement s'écrit :

$$
\underline{\underline{\sigma}}=\kappa \varepsilon_{V}(\mathbf{u}) \underline{\underline{I}}+2 \mu \underline{\underline{\varepsilon}}^{D}(\mathbf{u}) \quad \text { in } \Omega
$$

où $\varepsilon_{V}$ est la déformation volumique $\left(\varepsilon_{V}=\operatorname{div}(u)\right), \kappa$ est le module de compressibilité,

$$
\kappa=\frac{E}{3(1-2 \nu)}
$$

et $\underline{\underline{\varepsilon}}^{D}$ est la partie déviatorique de la déformation :

$$
\underline{\underline{\varepsilon}}^{D}=\underline{\underline{\varepsilon}}-\frac{\varepsilon_{V}}{3} \underline{\underline{I}}
$$

Quand le matériau devient incompressible, le module de compressibilité tend vers l'infini. Cela signifie que $\varepsilon_{V}$ doit tendre vers zéro pour vérifier la condition [2].

$$
\varepsilon_{V}=\operatorname{div}(\mathbf{u}) \longrightarrow 0 \text { quand } \nu \rightarrow 0.5
$$


La formulation forte [1] est aussi équivalente à la stationnarité d'un potentiel ПI qui, pour une formulation en déplacement s'écrit :

$$
\Pi(\mathbf{u})=\frac{1}{2} \int_{\Omega} \underline{\underline{\varepsilon}}(\mathbf{u}): \underline{\underline{\underline{\mathcal{C}}}}: \underline{\underline{\varepsilon}}(\mathbf{u}) d \Omega-\int_{\Omega} \mathbf{u} \cdot \mathbf{b} d \Omega-\int_{\partial \Omega_{t}} \mathbf{u} \cdot \mathbf{T}_{\mathbf{d}} d \Gamma
$$

Afin de modéliser des problèmes incompressibles ou quasi incompressibles, on considère un principe à 2 champs en introduisant une deuxième variable (la pression hydrostatique $p$ ) dans [7] :

$$
p=-\kappa \varepsilon_{V}(\mathbf{u})=-\frac{1}{3} \operatorname{Tr}(\underline{\underline{\sigma}})
$$

Quand $\kappa$ augmente, la déformation volumique $\varepsilon_{V}$ diminue et devient très petite. En incompressibilité totale, le module de compressibilité est infini, la déformation volumique est nulle (la pression reste finie, de l'ordre du vecteur traction appliqué).

La contrainte s'exprime par :
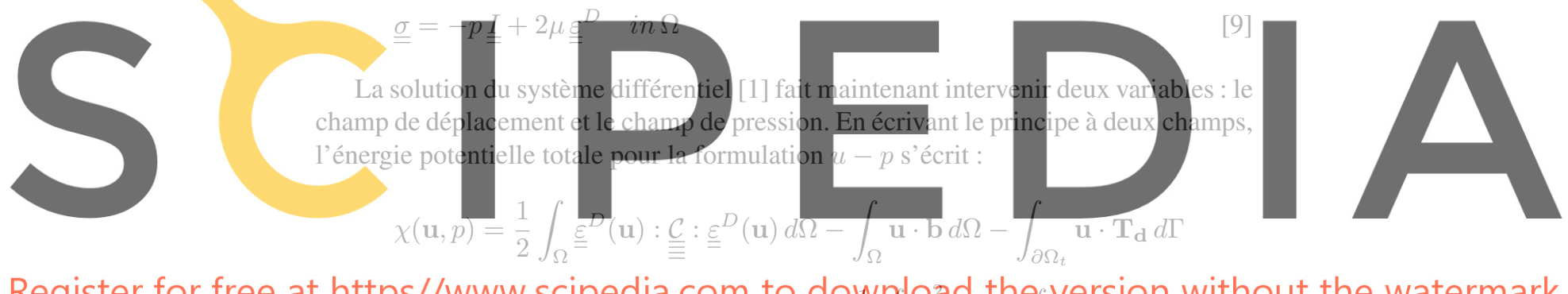

Register for free at https//www.scipedia.com to downlqad the version without the watermark

En écrivant la stationnarité de $\chi(\mathbf{u}, p)$ par rapport aux deux variables indépendantes $\mathbf{u}$ et $p$, on obtient :

$$
\begin{aligned}
& \int_{\Omega} \delta \underline{\underline{\varepsilon}}^{D}: \underline{\underline{\underline{\underline{\mathcal{C}}}}}: \underline{\underline{\varepsilon}}^{D} d \Omega-\int_{\Omega} p \delta \varepsilon_{V} d \Omega=R(\delta \mathbf{v}) \\
& -\int_{\Omega}\left(\frac{p}{\kappa}+\varepsilon_{V}\right) \delta p d \Omega=0
\end{aligned}
$$

où $R(\delta \mathbf{v})$ représente le travail virtuel des forces extérieures.

\subsection{Stabilité des formulations mixtes}

\subsubsection{Condition analytique de stabilité - la condition inf-sup}

La discrétisation de l'équation [11] donne lieu à un système élément finis du type (voir section 3) :

$$
\left(\begin{array}{cc}
\mathbf{K}_{\mathbf{u u}} & \mathbf{K}_{\mathbf{u p}} \\
\mathbf{K}_{\mathbf{u p}} T & \frac{1}{\kappa} \mathbf{K}_{\mathbf{p p}}
\end{array}\right)\left(\begin{array}{c}
\mathbf{u} \\
\mathbf{p}
\end{array}\right)=\left(\begin{array}{c}
\mathbf{f} \\
\mathbf{0}
\end{array}\right)
$$


La question est de savoir sous quelles conditions ce système d'équations est régulier. On peut montrer que c'est le cas (dans le cas de l'incompressibilité totale quand $\kappa \mapsto \infty$ ), pourvu que le noyau de $\mathbf{K}_{\text {up }}$ est vide. Plus précisément, l'interpolation déplacement-pression doit vérifier la condition de compatibilité LadyzhenskayaBabuška-Brezzi (Brezzi et Fortin 1991), aussi nommée condition LBB (ou inf-sup). Cette condition montre que les champs de déplacement et de pression ne peuvent être choisis séparément. La condition suffisante qui relie ces espaces (inf-sup ou LBB condition) s'écrit :

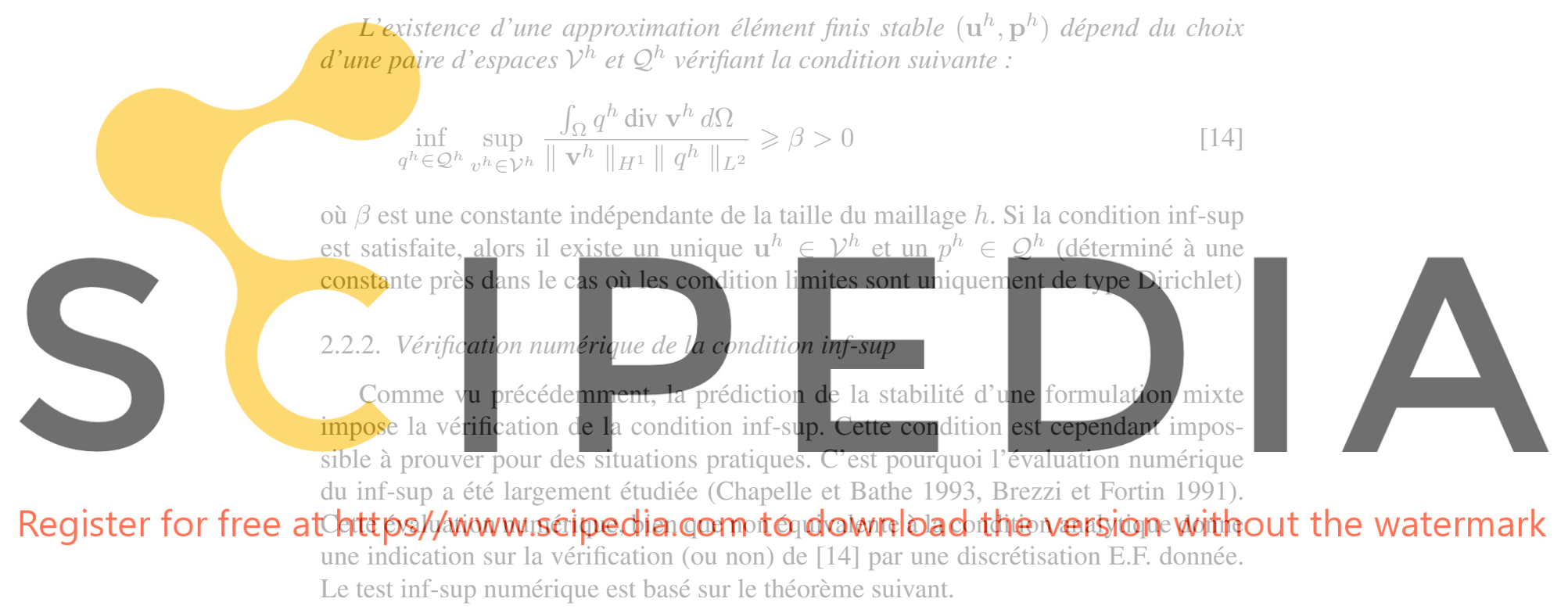

Proposition 1 Soient les matrices $\mathbf{M}_{\mathbf{u u}}$ et $\mathbf{M}_{\mathbf{p p}}$, associées respectivement aux produits scalaires dans $\mathcal{V}^{h}$ (norme $H^{1}$ ) et $\mathcal{Q}^{h}$ (norme $L^{2}$ ) et soit $\mu_{\text {min }}$ la plus petite valeur propre non nulle du problème aux valeurs propres suivant :

$$
\mathbf{K}_{\mathbf{u p}}{ }^{T} \mathbf{M}_{\mathbf{u u}}{ }^{-1} \mathbf{K}_{\mathbf{u p}} \mathbf{v}=\mu^{2} \mathbf{M}_{\mathbf{p p}} \mathbf{v}
$$

alors, la valeur de $\beta$ est simplement $\mu_{m i n}$.

La démonstration peut être trouvée dans (Malkus 1981) ou (Brezzi et Fortin 1991). Le test numérique proposé dans (Chapelle et Bathe 1993) consiste à tester une formulation mixte en calculant $\beta$ avec des maillages de plus en plus raffinés (Remarque : dans ce travail, on suivra la démarche présentée dans (Chapelle et Bathe 1993), et on considérera $\mathbf{S}_{\mathbf{u u}}=\int_{\Omega} \underline{\nabla} \mathbf{u}: \underline{\nabla} \mathbf{u} d \Omega$ au lieu de $\mathbf{M}_{\mathbf{u u}}$ dans [15)]. Sur la base de trois ou quatre résultats, on peut prédire si la valeur du inf-sup est bornée ou au contraire tend vers zéro quand le maillage est raffiné. La fiabilité de ce test est démontrée sur de nombreux exemples d'éléments en élasticité incompressible dans (Chapelle et Bathe 1993). 
Ce test est appliqué en considérant une séquence de maillages successivement raffinés. L'objectif est de contrôler la valeur du inf-sup, $\beta_{h}$ quand $h$ diminue. $\operatorname{Si} \log \left(\beta_{h}\right)$ décroît constamment lorsque $h$ tend vers zéro, on prédit que l'élément viole la condition inf-sup. Mais si $\log \left(\beta_{h}\right)$ se stabilise lorsque le nombre d'éléments augmente, alors le test numérique est vérifié.

\section{Diccrétication avec X-FEM}

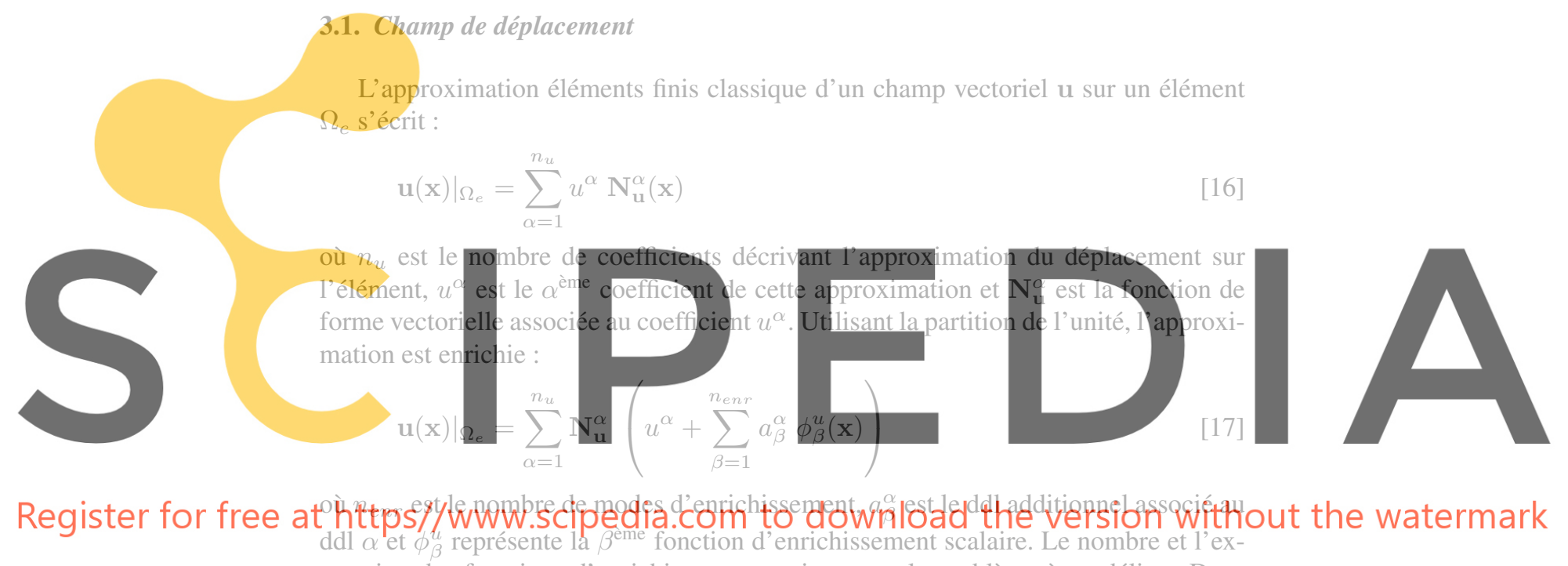

pression des fonctions d'enrichissement varient avec le problème à modéliser. Dans

le cas des trous (voir section suivante), aucun enrichissement n'est utilisé et seule la procédure d'intégration est modifiée. Le lecteur intéressé par le cas des inclusions matérielles et de la fissuration de matériaux incompressibles peut se référer à (Legrain et al. 2005a).

\subsection{Champ de pression}

En utilisant le même formalisme, l'approximation du champ de pression s'écrit :

$$
\left.p(\mathbf{x})\right|_{\Omega_{e}}=\sum_{\alpha=1}^{n_{p}} N_{p}^{\alpha}\left(p^{\alpha}+a^{\alpha} \phi^{p}(\mathbf{x})\right)
$$

où $n_{p}$ est le nombre de coefficients décrivant l'approximation sur l'élément, $p^{\alpha}$ est le $\alpha^{\text {ème }}$ coefficient de cette approximation, $N_{p}^{\alpha}$ est la fonction de forme scalaire associée au coefficients $\alpha$ et $\phi^{p}$ représente la fonction d'enrichissement scalaire du champ de pression.

Le but est ici de déterminer si la prise en compte de trous par X-FEM dans le cas de formulations incompressibles converge optimalement et passe la condition inf-sup. 


\subsection{Eléments finis mixtes stables}

Dans ce travail, nous nous intéressons à deux éléments finis mixtes stables : l'élément Mini (figure 2) introduit par Arnold, Brezzi et Fortin (Arnold et al. 1984) en considérant un champ de déplacement linéaire enrichi avec une fonction bulle, et un champ de pression linéaire (élément $P_{1}^{+}, P_{1}$ ). Le deuxième élément considéré est le T6T3 (élément $P_{2}, P_{1}$ ) (figure 2) qui est un élément fini mixte classique.

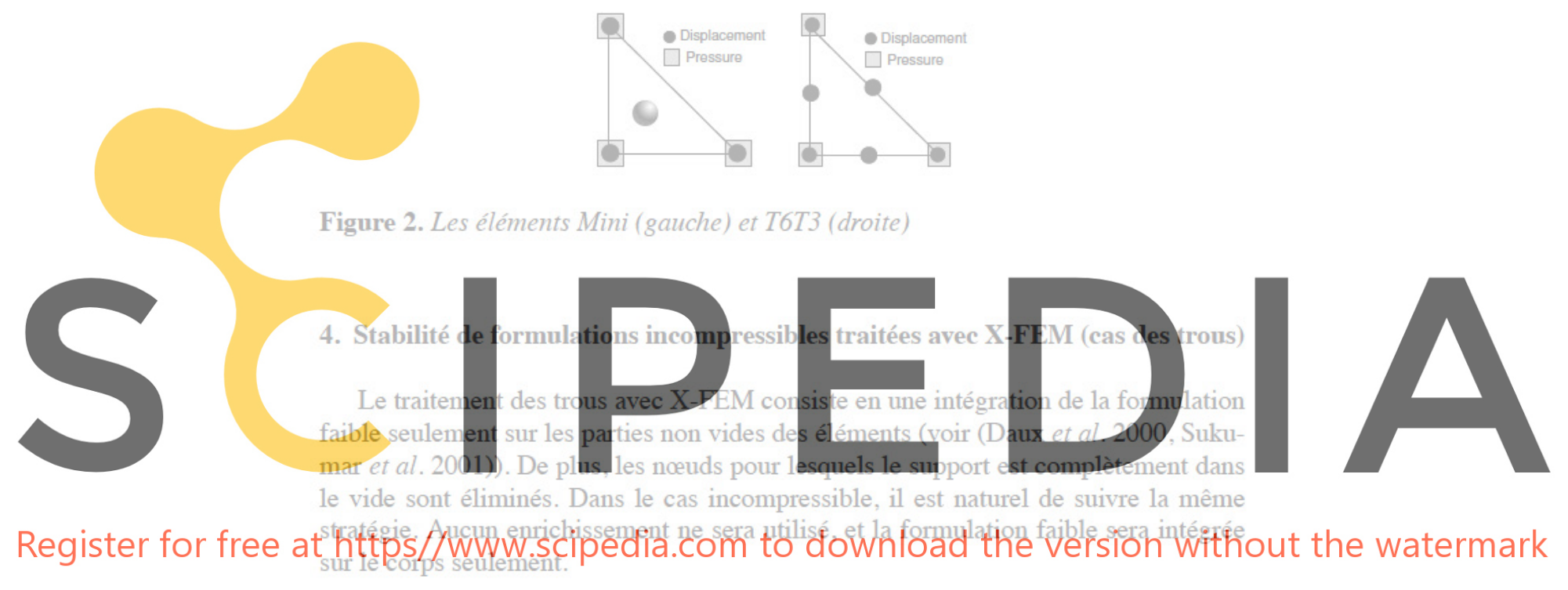

\subsection{Etude de convergence}

On considère une plaque infinie avec un trou circulaire sous traction uniaxiale. La solution exacte de ce problème est donnée dans (Sukumar et al. 2001). On considère un domaine carré de côté 2 avec un trou circulaire de rayon $a=0.4$ en son centre. Les tractions exactes sont imposées sur la frontière du domaine, et les mouvements de corps rigide sont bloqués. Le coefficient de Poisson est choisi égal à 0.5 (incompressibilité totale), et le module d'Young à 1.0. On suppose de plus l'état de déformation plane. La séquence de maillages utilisée pour l'étude de convergence est non structurée et ne tient pas compte du trou. L'erreur sur le champ de déplacement est mesurée par une erreur en énergie, et l'erreur sur la pression par une norme $L^{2}$. L'étude de convergence est réalisée avec les éléments Mini et T6T3. Les résultats sont présentés sur la figure 3 pour la pression et le déplacement. Comme illustré sur la figure 3 , le taux de convergence pour le déplacement et la pression sont similaires aux taux théoriques. Cela montre que cette stratégie préserve les taux de convergence éléments finis théoriques des éléments Mini et T6T3. 


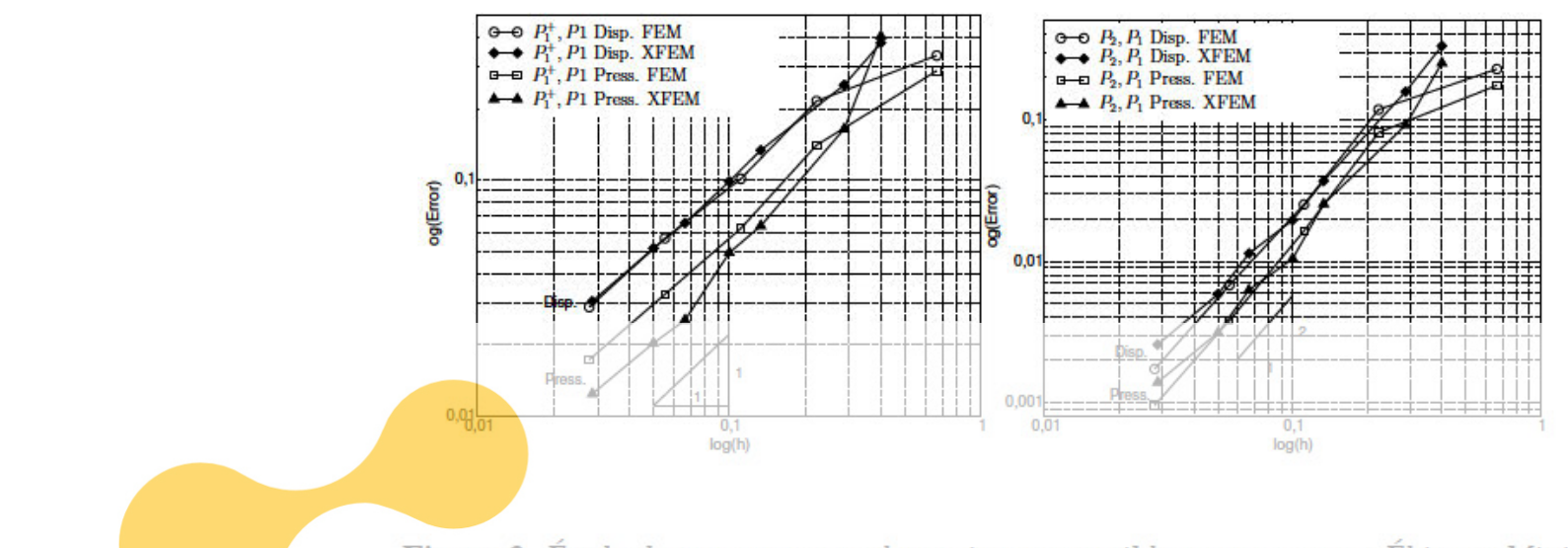

Figure 3. Étude de convergence, plaque incompressible avec un trou : Élément Mini (gauche) et T6T3 (droite)
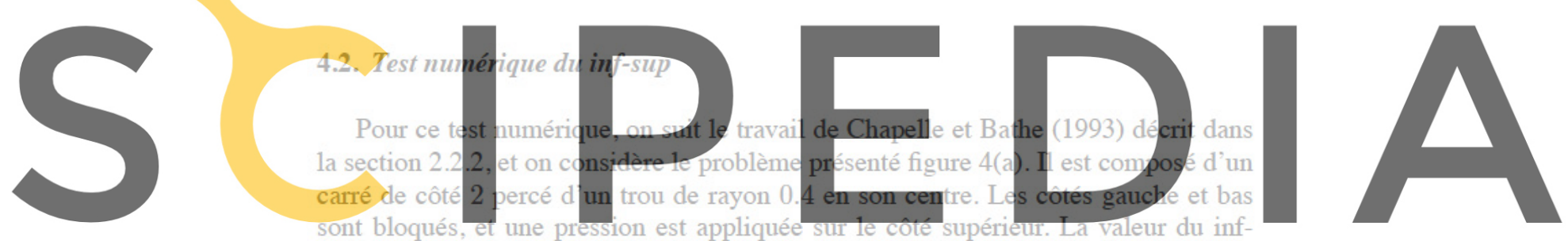

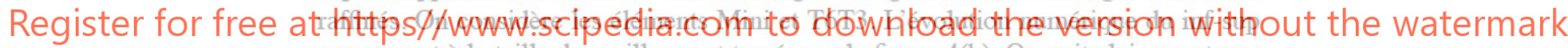
par rapport à la taille du maillage est tracée sur la figure 4(b). On voit clairement que l'inf-sup numérique est stable tant pour le Mini que pour le T6T3.

\subsection{Cavité carrée}

L'exemple suivant est adapté d'un test classiquement utilisé en mécanique des fluides pour quantifier la robustesse des éléments finis mixtes. Le problème est constitué d'une plaque carrée de côté 2 contentant un trou circulaire de rayon $a=0.4$ en son centre (voir figure 5(a)). Les faces supérieures, gauches et droites sont encastrées, alors que le déplacement vertical est bloqué sur la face supérieure. Le chargement est constitué d'un effort horizontal réparti sur la face supérieure. Les comportements des formulations mixtes FEM et X-FEM sont comparés avec leurs homologues en déplacement. Pour cela, on considère quatre formulations (FEM, X-FEM, mixtes ou non), et on fait varier le coefficient de poisson de 0.3 à 0.5 . On trace sur la figure 5(b) le déplacement maximum sur la ligne supérieure en fonction du module de compressibilité du matériau. Toutes les formulations en déplacement verrouillent comme en attestent les courbes de déplacement qui tendent vers zéro. A l'opposé, les formulations mixtes (FEM ou X-FEM) restent insensibles aux variations du module de compressibilité. 


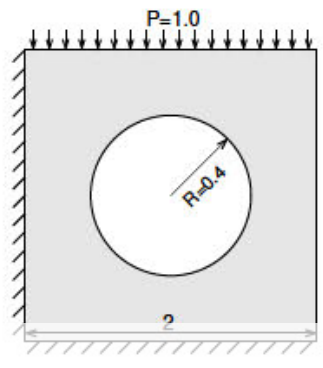

(a)

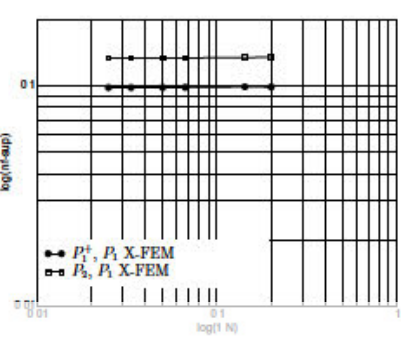

(b)

Figure 4. Évaluation du inf-sup pour les trous : (a) problème de référence, (b) Évolution du inf-sup numérique
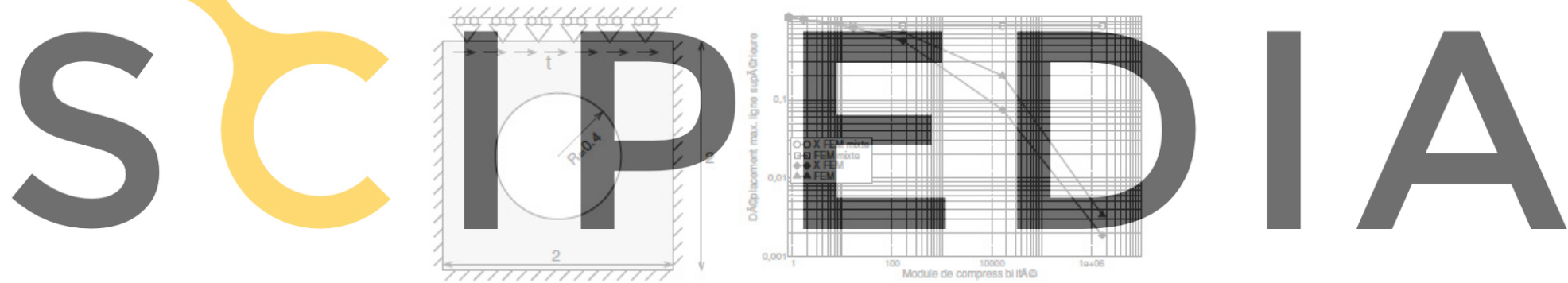

Register for free at https//www.scipedia.com to downloaid the version without the watermark

Figure 5. (a) Problème de cavité, (b) Évolution du déplacement sur la ligne supérieure en fonction du module de compressibilité

\section{Conclusion}

L'utilisation de la méthode X-FEM avec des formulations incompressibles a été présentée dans le cas des trous. Plusieurs validations ont été présentées afin de montrer que les taux de convergence optimaux de ces formulations sont conservés après enrichissement, et que leur tolérance au verrouillage est conservée. De plus, la vérification du critère inf-sup a été montrée numériquement, ce qui valide l'utilisation de ce type de formulations. Le cas des interfaces matériaux et de la fissuration (ainsi que d'autres exemples sur les trous) est traité dans une autre contribution (Legrain $e t$ al. 2005a). 


\section{Bibliographie}

Areias P. M. A., Belytschko T., « Non-linear analysis of shells with arbitrary evolving cracks using XFEM », International Journal for Numerical Methods in Engineering, vol. 62, p. 384-415, 2005.

Arnold D., Brezzi F., Fortin M., « Stable finite element for stokes equations », Calcolo, vol. 21, p. 337-344, 1984.

Brezzi F., Fortin M., Mixed and Hybrid Finite Element Methods, Springer, New York, 1991.

Chapelle D., Bathe K., « The inf-sup test », Computers \& structures, vol. 47, p. 537-545, 1993.

Chessa J., Belytschko T., « An extended finite element method for two-phase fluids », Journal of Applied Mechanics (ASME), vol. 70, p. 10-17, 2003.

Daux C., Moës N., Dolbow J., Sukumar N., Belytschko T., « Arbitrary branched and intersecting cracks with the eXtended Finite Element Method », International Journal for Numerical Methods in Engineering, vol. 48, p. 1741-1760, 2000.

Dolbow J., Belytschko T., « Volumetric locking in the element free Galerkin method », Int. J. Numer. Methods Eng., vol. 46, p. 925942, 1999.

Dolbow J., Devan A., « Enrichment of Enhanced Assumed Strain Approximations for representing Strong Discontinuities : Addressing Volumetric Incompressibility and the Discontinuous Patch Test », International journal for numerical methods in engineering, vol. 59, p. 47-67, 2004.

Dolbow J., Moës N., Belytschko T., « Modeling fracture in Mindlin-Reissner plates with the eXtended finite element method », Int. J. Solids Structures, vol. 37, p. 7161-7183, 2000.

Gravouil A., Moës N., Belytschko T., « Non-planar 3D crack growth by the extended finite element and level sets. Part II : level set update », International Journal for Numerical Methods in Engineering, vol. 53, p. 2569-2586, 2002.

Huerta A., Fernández-Méndez S., « Enrichment and Coupling of the Finite Element and Meshless Methods », International Journal for Numerical Methods in Engineering, vol. 48, p. 1615-1636, 2000.

Huerta A., Fernández-Méndez S., «Locking in the incompressible limit for the element free galerkin method », international journal for numerical methods in engineering, vol. 51, p. 1361-1383, 2001.

Huerta A., Vidal Y., Villon P., « Pseudo-divergence-free element free Galerkin method for incompressible fluid flow », Computer Methods in Applied Mechanics and Engineering, vol. 193, p. 1119-1136, 2004.

Hughes T., «Generalization of selective reduced integration procedures to anisotropic and nonlinear media. », International Journal for Numerical Methods in Engineering, vol. 15, p. $1413-1418,1980$.

Hughes T., Taylor R., Levy J., High Reynolds number, steady, incompressible flows by a finite element method, John Wiley \& Sons, 1978.

Legrain G., Moës N., Huerta A., « Stability of incompressible formulations enriched with XFEM », Computer Meth. Appl. Mech. Engrg., 2005a. Submitted.

Legrain G., Moës N., Verron E., «Stress analysis around crack tips in finite strain problems using the eXtended Finite Element Method », International Journal for Numerical Methods in Engineering, vol. 63, p. 290-314, 2005 b. 
Malkus D. S., «Eigenproblems associated with the discrete LBB condition for incompressible finite elements », Int. J. Eng. Sci., vol. 19, p. 1299-1310, 1981.

Melenk J., Babuška I., « The partition of unity finite element method : Basic theory and applications », Comput. Methods Appl. Mech. Engrg., vol. 139, p. 289-314, 1996.

Moës N., Belytschko T., « Extended Finite Element Method for Cohesive Crack Growth », Engineering Fracture Mechanics, vol. 69, p. 813-834, 2002a.

Moës N., Cloirec M., Cartraud P., Remacle J.-F., « A computational approach to handle complex microstructure geometries », Comp. Meth. in Applied Mech. and Engrg., vol. 192, p. 31633177, 2003.

Moës N., Dolbow J., Belytschko T., « A finite element method for crack growth without remeshing », International Journal for Numerical Methods in Engineering, vol. 46, p. 131-150, 1999.

Moës N., Gravouil A., Belytschko T., « Non-planar 3D crack growth by the extended finite element and level sets. Part I : Mechanical model », International Journal for Numerical Methods in Engineering, vol. 53, p. 2549-2568, $2002 \mathrm{~b}$.

Naylor D., « Stress in nearly incompressible materials for finite elements with application to the calculation of excess pore pressure. ", international journal for numerical methods in engineering, vol. 8, p. 443-460, 1974.

Réthoré J., Gravouil A., Combescure A., « An energy-conserving scheme for dynamic crack growth using the eXtended finite element method », International Journal for Numerical Methods in Engineering, vol. 63, p. 631-659, 2005.

Simo J., Rifai M., « A class of mixed assumed strain methods and the method of incompatible modes », International Journal for Numerical Methods in Engineering, vol. 29, p. $15951638,1990$.

Sukumar N., Chopp D. L., Moës N., Belytschko T., « Modeling Holes and Inclusions by Level Sets in the Extended Finite Element Method », Comp. Meth. in Applied Mech. and Engrg., vol. 190, p. 6183-6200, 2001.

Taylor R., Beresford P., Wilson E., « A nonconforming element for stress analysis », International Journal for Numerical Methods in Engineering, vol. 10, p. 1211-1219, 1976.

Wagner G., Moës N., Liu W., Belytschko T., « The Extended Finite Element Method for Stokes Flow Past Rigid Cylinders », International Journal for Numerical Methods in Engineering, vol. 51, p. 293-313, 2001. 